\title{
Normal Distribution Random Number Simulation Application
}

\author{
Lei Wang \\ College of Marine Engineering, Northwestern \\ Polytechnical University, Xi'an, China \\ Dept. of Naval Gun, Dalian Naval Academy, Dalian, \\ China \\ E-mail: spriter_007@163.com
}

\author{
Zheng-Guo Liu \\ College of Marine Engineering, Northwestern \\ Polytechnical University, Xi’an, China
}

\begin{abstract}
It was difficult to display the radar receiver noise realistically in radar simulated trainer. Analyzed the engender principle and characteristic of radar receiver noise. According to the method of changing uniformly distributed pseudorandom numbers to normally distributed pseudorandom numbers, put forward the calculation step of engender normally distributed pseudorandom numbers. And build the simulated model of radar receiver noise based on normally distributed pseudorandom numbers. Actualized the display function of radar simulated trainer and the display was right. The result of simulation indicated that the simulated model was feasibility, and increased the display fidelity of radar simulated trainer effectively.
\end{abstract}

Keywords-normal distribution; radar receiver; application; simulating

\section{INTRODUCTION}

In the ship-borne radar simulation training equipment in order to make more realistic target simulation environment, radar receiver noise, meteorological and ground clutter simulation is needed. Receiver clutter noise is inevitable, existing various working conditions. Receiver noise cluttered display range is small, when the target distance is far, ECHO will not be under the influence of receiver noise and clutter, and therefore does not affect the detection, identification and tracking. However, when the target distance is close, especially for anti-ship missile radar, such as the smaller, weaker echoes, finding distance, higher risk will echo submerged inside the receiver noise clutter, the detection, identification and tracking caused a great deal of influence. To train operator skills, creating relatively realistic radar simulation training equipment must return environment, improved radar operator for anti-ship missile target recognition capability. Therefore, the receiver noise simulation of radar Simulator fidelity is essential work.

Simulation of ship-borne radar function simulation and video signal simulation method, video signal simulation using signal phase relationship-driven simulation training equipment, so the video signal simulation more complex than functional simulation, features stronger, higher fidelity, but complex, runs slower. Functional simulation is based on the principles of clutter, through relatively simple model in radar simulation equipment shows on the display-related interference noise and clutter and making them affect the observation, identification, tracking, and so on, so as to meet the requirements of radar simulation shows the fidelity and speed. References [1-8] using different methods of radar sea clutter simulation models are discussed, but less discussed in clutter simulation of radar receiver noise. References [9] the perspective of signal describes the simulation of radar receiver noise. In the actual radar simulation training equipment, used in rectangular area or in a circular area to trace the way the receiver noise display simulation, the effect is not real, cannot meet the requirements of training in complex electromagnetic environments. Based on the simulation of ship-borne radar training requirements, mainly from the perspective of functional simulation build receiver noise display model, and as a follow-up to the creation of realistic work environment.

\section{PRINCIPLES OF RADAR RECEIVER NOISE}

Includes external antenna of radar receiver noise and when internal noise of the receiver. External antenna noise covers a surface of the Earth launch of radio noise, atmospheric radiation, cosmic background radiation noise radio noise. Typically, external noise power antenna equivalent noise temperature of an antenna.

$$
P_{\mathrm{a}}=k \cdot T_{\mathrm{a}} \cdot B_{R}
$$

In the equation, $k=1.38 \times 10^{-23} \mathrm{~J} / \mathrm{K}$ is Boltzmann constant, $T_{\mathrm{a}}$ is equivalent noise temperature of an antenna $(K), B_{R}$ is instantaneous radar receiver bandwidth.

Internal noise is measured by the noise figure of the receiver $\left(N_{F}\right)$, it is defined as the actual output of the receiver noise power receiver and ideal ratio of the output noise power. Usually, the noise figure of range is $0 d B<N_{F}<15 d B$. External antenna noise relative to the receiver noise is much narrower, in simulation, you can ignore[10]. Here we only discuss the principles and characteristics of internal radar receiver noise.

We commonly think of white noise is the mean value is zero, the spectral density of the normal number of stationary stochastic processes, it can be described as below.

$$
S_{X}(\omega)=S_{0},-\infty<\omega<+\infty\left(S_{0}>0\right)
$$

White noise can be defined as the mean value is zero, the autocorrelation function is a function of stochastic process, and in the process when $\mathrm{t}_{1} \neq \mathrm{t}_{2}, \mathrm{X}\left(\mathrm{t}_{1}\right)$ and $\mathrm{X}\left(\mathrm{t}_{2}\right)$ are not related. 
White noise is an idealized mathematical model. In practical engineering application, if a noise actually consider much more useful frequency band range is relatively "flat" spectral density, you can approximate it as white noise to deal with. According to this characteristic you can think of radar receiver noise is white noise and can thought to be subject to the standard normal distribution to approximate the Gaussian noise[11].

From this, it can be a very important conclusion: Radar receiver noise can be expressed as a subject with mean 0 , normal distribution of variance $\delta$ random process.

\section{SIMULATION OF RADAR RECEIVER NOISE MODEL}

Through analysis we know of radar receiver noise can be expressed as a normally distributed stochastic processes. However, commonly used in computer simulation language can directly generate uniformly distributed pseudorandom numbers, other probability distribution of random numbers only in the uniform distribution on the basis of production. Arbitrary random variables has a certain relationship with evenly distributed and normally distributed random variables can be uniformly distributed random numbers obtained through appropriate transformations.

\section{A. Normal Distribution Random Number Generation Method}

Using approximate sampling can also generate standard normal distribution random variable. Use the central limit theorem in changing uniform random variable distribution function to the standard normal distribution. According to the central limit theorem, find sum(x) of several unrelated random numbers $\left(x_{1}, x_{2}, \cdots, x_{n}\right)$.

$$
x=\sum_{i=1}^{n} x_{i}
$$

If $\mathrm{n}$ is big enough, then $\mathrm{x}$ is normal distribution random variables. Generally, $n=12$, mean of the sum of independent random variable is equal to the sum of random variables mean, Sum of independent random variables such as the sum of the variance of random variable. Shows as:

$$
\begin{gathered}
E(x)=E\left(x_{1}\right)+E\left(x_{2}\right)+\cdots E\left(x_{n}\right) \\
\sigma_{x}^{2}=\sigma_{x_{1}}^{2}+\sigma_{x_{2}}^{2}+\cdots \sigma_{x_{n}}^{2}
\end{gathered}
$$

From the equation (4) and (5), we can know that the mean of $x$ is 0 , and the variance of $x$ is 1 , and $x$ is normally distributed variable. This algorithm is also known as the 12 summation method.

\section{B. Simulation Model Of Radar Receiver Noise Power}

Radar receiver noise power depends on the receiver's signal to noise ratio, performance parameters such as sensitivity, but decided the noise and clutter on the radar display shows range and brightness. According to the radar machine noises characteristics of instantaneous power generated by a uniformly distributed random numbers using the Central limit theorem method to generate normal random number sequence, the established radar receiver noise simulation model of instantaneous power $(\mathrm{P})$.

$$
P_{i n}=\frac{1}{2} \sigma_{n}^{2}\left[\left(\sum_{i=1}^{12} x_{i}-6\right)^{2}+\left(\sum_{j=1}^{12} y_{j}-6\right)^{2}\right]
$$

In the equation, $x_{i}(i=1,2, \cdots, 12)$ and $y_{j}(j=1,2, \cdots, 12)$ both are uniform distribution independent random variables. Variables $\left(\sum_{i=1}^{12} x_{i}-6\right)^{2}$ and $\left(\sum_{j=1}^{12} y_{j}-6\right)^{2}$ both are standard normal distribution random variables.

The receiver noise power determined by the size of the noise and clutter on the radar display of the display range and brightness, in the range of simulation needs and radar combined.

\section{RECEIVER NOISE DISPLAY SIMULATION}

\section{A. Receiver Noise Power Spectrum Simulation}

According to 3.2 in the radar receiver power simulation model, simulation algorithms are as follows:

First of all, by generating uniformly distributed random number which taking value interval is $\left(0,2^{k}-1\right), \mathrm{k}$ is half the length of computer word, most of the current computer is a 32-bit, so $\mathrm{k}=16$.

Second, nominal change. Change uniform distribution random variables range in $(0,1)$.

Third, mean value shifting. Taking value interval change into $(-0.5,0.5)$, the mean value is $E(x)=0$ and variance is $\sigma^{2}=1 / 12$.

Fourth, According to the central limit theorem, find sum (x) of several unrelated random numbers $\left(x_{1}, x_{2}, \cdots, x_{n}\right)$.

Fifth, according to the formula (9) draws an image.

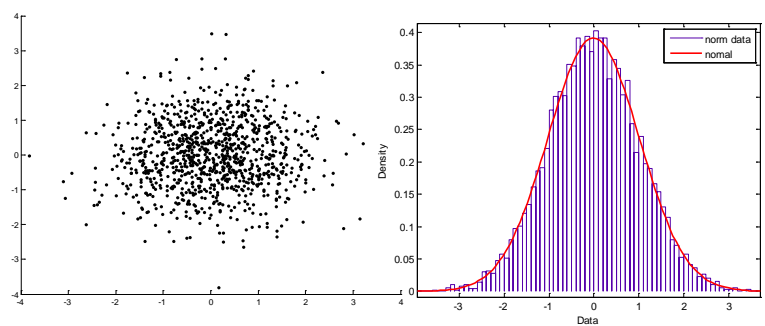

Figure 1 Distribution of radar receiver noise clutter and power 


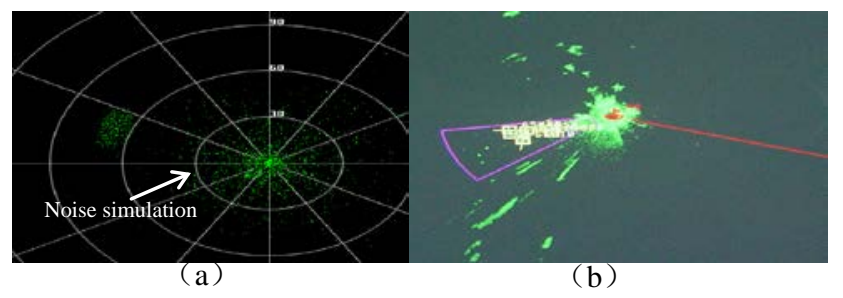

Figure 2 Radar noise simulation shows.

It generates 10,800 random numbers of standard normal distribution. These random numbers distribution and power spectrum shows as shown in Fig.1, in line with the distribution of radar receiver noise power.

\section{B. Receiver Noise Display Simulation}

After radar connected high voltage, the radar waves in the receiver receives is displayed on the monitor. These scattered wave contains not only a useful target, also includes the external surface, ground and weather clutters, these external clutter, ECHO noise clutter and receiver at the same time shows on the display, together form the radar display. Receiver noise simulation of radar training Simulator displays the process described below.

1) Receiver noise model: Based on normal distribution characteristics, mean values $(\mu)$ determine the symmetry centers of the normal distribution, and mean square error $(\sigma)$ determines the dispersion of normal distribution. In simulation, let $\mu=0$, line $x=0$ is symmetry axis of Normal distribution random number. Mean-square $(\sigma)$ determined by radar display range, therefore, the intensity of noise change with the radar range. Simulate radar PPI display receiver noise by drawing a point way. To determine Cartesian coordinates of noise display.

$$
\left\{\begin{array}{l}
x=O_{x}+l \cdot(n \backslash r) \cdot \sin \theta \\
y=O_{y}+l \cdot(n \backslash r) \cdot \cos \theta
\end{array}\right.
$$

In the equation, point $\left(O_{x}, O_{y}\right)$ is radar simulation screen center, $r$ is the radius of a radar simulator screen, $l$ is radar display range, $n$ is normal distribution random number. In order to avoid noise displayed out of the screen, to limit the normal distribution of random numbers, the method is screen radius $(r)$ complementation. $\theta$ is receiver noise display angle, also is the polar angle of radar scanning ling. The polar angle is determined by the radar scanning speed.

2) Simulation: For example, a certain type of ship-borne radar plan position indicator (PPI) as an example of the simulation. Simulation monitor 19-inch widescreen LCD monitor, resolution to $1280 \times 1024,32$-bit true color, computer CPU is P4 2.4GHz RAM 2GB, type Radeon 7000 graphics chips, memory is $256 \mathrm{MB}$ using the VC6.0++ program. Request simulation effect of realistic, programs run smoothly, there must be no Cottonwood.
Simulation plan position indicator (PPI) center screen pixel coordinates is $O(432,499)$, screen radius is $r=379$ pixel. In accordance with the calculation method described in 4.1, each radar scan line position 30 on the normal distribution of random numbers, scan the entire circumference of a total of 10,800 noises. Radar display initial range is set to $120 \mathrm{Km}$. Set speed of radar antenna is 30 degrees/sec. Both sides of the scan line 0.5 degree determine the scope display for noise. According to the formula (10) converted to normal random number generation $\mathrm{o}$ the rectangular coordinates of the Centre, by way of calling $\mathrm{C}++$ drawing functions to draw in the simulation screen mapped. Results in Fig.2 (a) as shown.

By changing radar display range, then change the standard deviation of the normal distribution random number, thus changing the normal distribution random number discrete degree. Can generate a range of changes, the radar receiver noise on an analog monitor shows the effect of scope change.

3) Simulated effect analysis: Simulations are able to realistically display of radar receiver noise, whether the program runs smoothly and whether they can meet the needs of radar training simulation equipment used simulation models for testing the effectiveness of standards. Fig.2 (b) is a certain type of $3 \mathrm{~cm}$ actual radar receiver noise and sea clutter image. Through the analysis of Fig.2 (a), (b) valid for two simulation effect of realistic picture, simulation models with high availability.

\section{SUMMARY}

Analysis based on the principle of radar receiver noise in the receiver noise display simulation models, radar speed display and organic combination of radar display range to the receiver noise model, changed without receiver noise simulation of radar training Simulator, or poor results could not meet the requirements of radar simulation training in complex electromagnetic environments of the status quo. The receiver noise simulation of radar display model used in the simulation training system of a certain type of ship-borne radar, shows that this algorithm is able to meet the needs of radar function simulation, reach effective training to improve radar operator discovery, identification, tracking, the ability of the effect. Display of radar receiver noise simulation model is established in this paper can be used not only in PPI display, can also be applied to other types in the simulation of radar receiver noise displayed on the monitor.

\section{REFERENCES}

[1] Hu Jurong, Cao Ning, Lu hao. A general model of Radar Sea clutter simulation [J], computer applications and software, 2009, 26 (7): 175-177

[2] Fabrizio Berizzi, Enzo Dalle-Mese. Scattering from a 2-D sea fractal surface: fractal analysis of the scattered signal [J]. IEEE Trans. On A. \& P (S0018-926X), 2002, 50(7): 912-925.

[3] Zhang Guo, Lu Weihong, Tong Ningning. Weibul1 analysis and improvement of distribution clutter model [J]. a modern defense technology, 2010,38 (1): 109-112 
[4] Luo Le, Hu linhua. Simulation of jamming sea clutter characteristics [J]. Journal of air force radar College, 2008, 22 (4): 268-270

[5] Zou Huanxin, Chen zhenlin, Zhou Shilin, etc. Based on ZMNL compound k-distributed radar sea clutter simulation [J]. a modern radar, 2009,31 (2): 63-66

[6] Sun Yu. Spherically invariant stochastic process based simulation of coherent correlated k-distributed sea clutter [J]. Science, technology and engineering, 2009.19 (17) 5144-5147.

[7] Wan Zhou. Temporal-spatial correlated k-distributed sea clutter based on SIRP simulation [J], electronics. 2009, 37 (12): 2672-2676.
[8] Wen Ouyang, You He, Yu Jin. Based on statistics model of temporal-spatial correlated sea clutter simulation [J]. Journal of system simulation, 2006, 18 (2): 467-471.

[9] Shen Huifang, Lai Honghui. Modeling and simulation of radar coherent clutters [J]. Radar science and technology, 2009, 7 (6): 447-451.

[10] Qi Qiang, Fengjun Deng. Characteristics of radar receiver noise analysis [J], value engineering, 2012, 31 (18): 187-191

[11] Guoyu. W, Liandong Wang. Mathematical simulation and evaluation of radar electronic warfare system [M]. Beijing: national defense industry press, 2004, 6:180. 